\title{
EDITORIAL
}

\section{Making research more useful and impactful}

Enoka Corea (Dhttp://orcid.org/0000-0003-1450-2098, Himani Molligoda (Dhttp://orcid.org/0000-0002-9216-9035, Co-Editors

\section{Keywords: Research Impact}

(C) Authors. This is an open-access article distributed under a Creative Commons AttributionShare Alike 4.0 International License (CC BY-SA 4.0), which permits unrestricted use, distribution, and reproduction in any medium, provided the original author and source are attributed and materials are shared under the same license.

The purpose of medical research is to generate new knowledge that will advance understanding of human health and disease or lead to interventions that enhance human wellbeing. Every researcher hopes that the results of his/her research will improve the quality of life of the general public and have an impact on society and the economy. Unfortunately, much postgraduate research does not result in practical, implementable and generalizable knowledge and the only resulting outcomes are training of a few research students and some few local publications.

Even in the research institutes and universities, there are very few groups that work on public health problems for the extended length of time required to generate valid results. Funding bodies and institutions compound the problem by valuing short term successes and outputs such as presentation of abstracts, publication of interim reports or annual performance appraisal.

However, as research funding dries up, researchers are being requested to be more accountable and produce more societal benefits through research. So, what can researchers do to enhance the societal value and impact of your research and how does an individual researcher evaluate his/her research for usefulness and impact?

Let's look at how you, as a researcher, can increase the societal benefits and impact of your research. The impact of research could range from increased awareness to implementation in clinical practice and, ultimately, to patient benefit [1].

Firstly, identify the real purpose and long-term use of your research. Choose problems, interventions and outcomes that are important and relevant to patients and clinicians and the disease burden of the population in which you live. Identify and consult the end users/key beneficiaries of your research to determine their priorities and needs and tailor your research question and outcome measures accordingly.

Take a holistic view. Look at the whole picture and the long term, rather than the immediate goals of fund raising or publication. Research should be thought of as a journey of epic proportions rather than a series of short field trips.

Do a systematic and comprehensive global and local literature review and make contact with other institutions and researchers in the field to avoid duplication and to add value 
to your research. Your research should be built on a complete and thorough understanding of the existing evidence.

Get your research proposal critiqued for practical methods and usability during peer review, and not just for ability to generate new knowledge. Measure the cost effectiveness of your intervention during the study so that the potential user is aware of the costs.

Collaborate and engage with policy makers and people in the field, including government organisations, corporates and NGOs, to ensure that the results of your research are applied into practice rapidly and efficiently. Be proactive regarding research dissemination, targeting influential stakeholders early in a meaningful manner. Align your key message to what is most relevant to them.

Go deeper rather than broader. Every research project throws up more questions than it answers. Each piece of research should result in a series of spin off research projects, each seeking to advance knowledge in another related area of research. Each piece of research should add a piece to the solution of the jigsaw puzzle which is the research problem.

Secondly, let us look at how a researcher can evaluate his/her work for its potential social benefit and impact. This is a necessary skill for researchers to acquire as they may be called on to describe the impact of their work on many occasions. The funding agency may ask for a description of the societal benefit of the research in the grant application, the institution where the research was conducted may require it as part of the interim or final research report or it may be a prerequisite for publication demanded by journal editors.

A framework for the analysis of the social impact of research has been published [2]. This framework provides a useful set of descriptive categories that prompt the researcher to evaluate the impact of his/her research over a wide range of areas, from research related impacts to policy impacts, services impacts and societal impacts.

Research related impacts include publications, patents and citations but also the type of problem addressed, type of research method used including innovations, commercial potential and translatability, building up of research networks, leadership position obtained through the research such as membership of committees, editorships etc., educational use of research etc. Policy impacts may range from direct influence on policy to supportive evidence for existing potential policies. Research can impact on services by influencing evidence-based practice, quality of care and cost effectiveness. Research can also have societal impacts by changing attitudes and behaviours, improving equity and promoting sustainable development.

Academic institutions should work to enhance the societal benefits of research by assessing and rewarding researchers on the basis of the long-term impact of their research and by supporting the formation of longitudinal research collaboration networks. 
Journal of the Postgraduate Institute of Medicine 2019; 6 (1): E 92: 1-3

doi: http://dx.doi.org/10.4038/jpgim.8256

\section{References}

1. Anthony P. Weiss. Measuring the Impact of Medical Research: Moving From Outputs to Outcomes. American Journal of Psychiatry 2007 164:2, 206-214 https://doi.org/10.1176/ajp.2007.164.2.206

2. Kuruvilla S, Mays N, Pleasant A, et al. Describing the impact of health research: $a$ research impact framework. BMC Health Serv Res. 2006;6:134. doi: 10.1186/1472- $\Xi$ 6963-6-134. https://doi.org/10.1186/1472-6963-6-134 